\title{
Dynamics and nonequilibrium states in the Hamiltonian mean-field model: A closer look
}

\author{
Damián H. Zanette Æ $^{\Re}$ \\ Consejo Nacional de Investigaciones Científicas y Técnicas, \\ Centro Atómico Bariloche and Instituto Balseiro, \\ 8400 San Carlos de Bariloche, Río Negro, Argentina. \\ Marcelo A. Montemurrof \\ Facultad de Matemática, Astronomía y Física, Universidad Nacional \\ de Córdoba, Ciudad Universitaria, 5000 Córdoba, Argentina.
}

(Dated: October 24, 2018)

\begin{abstract}
We critically revisit the evidence for the existence of quasistationary states in the globally coupled XY (or Hamiltonian mean-field) model. A slow-relaxation regime at long times is clearly revealed by numerical realizations of the model, but no traces of quasistationarity are found during the earlier stages of the evolution. We point out the nonergodic properties of this system in the short-time range, which makes a standard statistical description unsuitable. New aspects of the evolution during the nonergodic regime, and of the energy distribution function in the final approach to equilibrium, are disclosed.
\end{abstract}

PACS numbers: 05.20.-y, 05.45.Pq, 05.70.Fh

The globally coupled version of the XY model, given by the Hamiltonian

$$
\mathcal{H}=K+V=\frac{1}{2} \sum_{i=1}^{N} p_{i}^{2}+\frac{1}{2 N} \sum_{i, j=1}^{N}\left[1-\cos \left(\theta_{i}-\theta_{j}\right)\right],
$$

has recently attracted considerable attention $11,2,3,6$, 国, 6, 6, 8, 9. This model, usually referred to as the Hamiltonian mean-field (HMF) model, describes an ensemble of $N$ rotators characterized by their angles $\theta_{i}$ and conjugate momenta $p_{i}$. In contrast to the usual XY model, interactions affect all pairs of rotators in the ensemble. The HMF model has been studied in connection with the emergence of collective self-organized states [1], dynamical behavior near phase transitions [2], and anomalous diffusion in phase space 迎, among other dynamical and thermodynamical aspects.

The canonical thermodynamical description of the HMF model can be completely carried out [1, 3]. In this description, it is useful to introduce the "magnetization" $\mathbf{M}=N^{-1} \sum_{i}\left(\cos \theta_{i}, \sin \theta_{i}\right) \equiv\left(M_{x}, M_{y}\right) \equiv M \exp (\mathrm{i} \Phi)$ and the average energy per particle $U=E / N$, with $E \equiv \mathcal{H}$. The canonical approach predicts that the values of the magnetization and the average energy at equilibrium, $M_{\mathrm{eq}}$ and $U_{\mathrm{eq}}$, are related by

$$
U_{\mathrm{eq}}=\frac{T}{2}+\frac{1}{2}\left(1-M_{\mathrm{eq}}^{2}\right)
$$

where $T$ is the canonical temperature. The system undergoes a ferromagnetic-like, second-order phase transition

\footnotetext{
*Electronic address: zanette@cab.cnea.gov.an

${ }^{\dagger}$ Electronic address: mmontemu@famaf.unc.edu.an
}

at $T_{c}=1 / 2$, from a state with $M_{\text {eq }} \neq 0$ at low temperatures to a state of vanishing magnetization at high temperatures. The energy at the transition is $U_{c}=3 / 4$. These predictions are mostly confirmed by the numerical solution of the HMF equations of motion at fixed total energy, i.e. in a microcanonical scenario. In this situation the equilibrium temperature is defined through the kinetic energy

$$
T_{\text {eq }}=\frac{2}{N} K_{\text {eq }},
$$

and $U=T_{\text {eq }} / 2+\left(1-M_{\text {eq }}^{2}\right) / 2[$ cf. Eq. (2) $)$.

Deviations between the canonical predictions and the microcanonical (numerical) results were however reported in the region of energies just below the critical point, $0.5<U<U_{c}$, where numerical realizations reveal extremely slow relaxation toward the asymptotic state 11, 2]. Such deviations, observed when the ensemble of rotators is prepared in specially chosen initial conditions - namely, the so called water-bag initial conditions - have been associated with the existence of long-lived states where, in the thermodynamical limit $N \rightarrow \infty$, the system would spend asymptotically long times. In that limit, canonical equilibrium would never be reached. It has been suggested that the HMF longlived states - which would replace canonical equilibrium in the thermodynamical limit - would be well described by the equilibrium distributions predicted by Tsallis's generalization of thermodynamics [6, 6, 10]. This generalization, in fact, relaxes the assumption of extensivity of the Boltzmann-Gibbs formulation, and is therefore presumably expected to describe the equilibrium statistics of systems with long-range interactions [11], as in the Hamiltonian (1).

Water-bag (WB) initial conditions fix $\theta_{i}=0$ for all $i$, and the momenta $p_{i}$ are chosen at random from a 
uniform distribution in such a way that $\sum_{i} p_{i}=0$ and $K=\sum_{i} p_{i}^{2} / 2=N U$. For these initial conditions, in fact, the potential energy vanishes. For a typical numerical realization at energies just below the critical point $U_{c}$, the dynamics of the HMF model can be summarized as follows. In the first stage, whose duration is essentially independent of the system size $N$ and equals a few time units [7], there is a rapid broadening of the distribution in $\theta$. This implies an abrupt growth of the potential energy from its initial value $V(t=0)=0$, to a value close to that predicted at canonical equilibrium, $V_{\text {eq }}=N\left(1-M_{\text {eq }}^{2}\right) / 2$. The kinetic energy drops accordingly, from $K(t=0)=N U$ to a value close to $K_{\text {eq }}=N T_{\text {eq }} / 2$, and the initial unbalance between $K$ and $V$ is drastically reduced. As explained below, the details of the evolution in this stage of kinetic relaxation depend strongly on the specific realization of the WB initial condition. Typically, however, the potential energy exceeds $V_{\text {eq }}$ and attains a maximum, while $K$ reaches a minimum. From then on, the system enters a stage of slow relaxation toward canonical equilibrium.

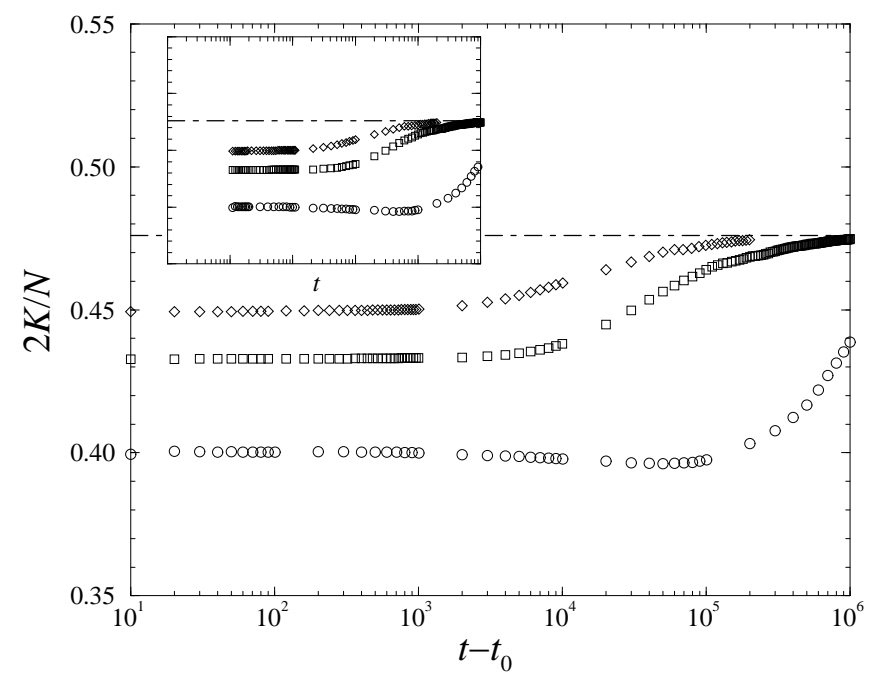

FIG. 1: Nonequilibrium temperature $T(t)=2 K(t) / N$ of the HMF model for $U=0.69$ and $N=500$ (diamonds), $10^{3}$ (squares), and $10^{4}$ (circles), from Fig. 1(b) of Ref. 6. Note that data are plotted against $t-t_{0}$, with $t_{0}=100$. The horizontal line indicates the canonical equilibrium temperature $T_{\text {eq }}=$ 0.476 . The inset shows the same data against $t$. The scales of the axis in the inset are the same as in the main plot.

The slow-relaxation regime of the HMF model was reported in the first systematic study of this system [1]. In Ref. 3 it was quantitatively characterized by analyzing the relaxation of the nonequilibrium entropy $S(t)$ toward its equilibrium value $S_{\text {eq }}$ as a function of $N$. The difference $\left|S(t)-S_{\text {eq }}\right|$ was shown to approach an asymptotic value of order $N^{-1 / 2}$ within a relaxation time $\tau_{r} \sim N$. The same scaling has recently been reported for the relaxation time of the nonequilibrium temperature, $T(t)=$ $2 K(t) / N[8]$. The states visited by the system during the slow-relaxation regime have been named quasistationary states, presumably by analogy with the quasiequilibria reported for one-dimensional gravitational systems 12, 13 and similar long-lived states in many-body systems with long-range interactions [14]. The name suggests that the time scales associated with the evolution of such states are much longer than those of any other regime during the whole evolution. However, the evolution of $S(t)$ toward $S_{\text {eq }}$ reveals no dynamical regime other than slow relaxation [3, 15].

Latora, Rapisarda and Tsallis (LRT), on the other hand, have claimed to demonstrate the entity of quasitationary states (QSS) from the analysis of the evolution of the nonequilibrium temperature $T(t)$ [6]. For clarity in the discussion, we reproduce in the main plot of Fig. 1 their figure 1(b) of Ref. 6, where $T(t)$ is plotted against time for several values of $N$, as obtained from numerical integration of the HMF equations of motion for $U=0.69$ [16. This value of the energy corresponds to the largest observed deviation of numerical results with respect to the canonical equilibrium prediction [1, 3. In the plot, the initial stage of kinetic relaxation is disregarded. For short times, we observe a wide plateau where $T(t)$ is apparently constant, but substantially differs from its equilibrium value. At long times, $T(t)$ has reached $T_{\text {eq }}$ and remains at equilibrium. These two plateaus are connected by a crossover stage where $T(t)$ exhibits an inflexion point and its variation is seemingly faster. Consequently, LRT conclude that the slow-relaxation regime consists in turn of a first stage where the system is "trapped" in a QSS, and a subsequent crossover toward canonical equilibrium. Since the position $\tau_{C}$ of the crossover shifts to larger times as $N$ grows, $\tau_{C} \sim N$, LRT deduce that in the thermodynamical limit the QSS will be observed at all times, and that canonical equilibrium will never be attained. As we argue in the following, however, the presence of the plateaus and, thus, the existence of a QSS in these specific realizations of the HMF model, is an artifact of the peculiar way chosen to plot the data in Fig. 1.

In the first place, in order to exclude the initial stage of kinetic relaxation, LRT choose to plot $T(t)$ as a function of $t-t_{0}$, with $t_{0}=100$, instead of simply cutting off the time axis at $t_{0}$. Such procedure, which would be innocuous in a linear time scale, has far-reaching consequences in the logarithmic scale of Fig. 1. In fact, the point $t=t_{0}$ becomes shifted to $-\infty$ in the logarithmic variable, which considerably enhances the impression of having a plateau at small $t$. In the inset of Fig. 1 we plot $T(t)$ against its original variable - also in logarithmic scale - showing that the short-time plateau shrinks by one decade. The existence of a crossover state joining the two plateaus is itself an illusion created by the logarithmic scale, even in the original variable $t$. Indeed, it can be easily shown that the graph of a function with monotonic first derivative can display an (otherwise inexistent) inflection point by the simple expedient of using a logarithmic scale in the horizontal axis 17].

In order to avoid these undesirable effects and to re- 
veal the true nature of the evolution of $T(t)$ in the slowrelaxation regime, we opt for using linear time scales. Figure 2 shows, plotted with diamonds, the same data as in Fig. 11 for $N=500$ up to $t=2500$, while the inset shows the whole temporal range. We see that there are no traces of a short-time plateau, except perhaps for a small interval around $t=500$. It might however be argued that the vertical scale in this plot has been exaggeratedly amplified. To decide over this point, we have used a Padé-like approximation to fit the data over the whole time range, shown as a curve in the inset. The quality of the fitting is quite acceptable. Its extension to the short-time range, also shown as a curve in the main plot, proves that in this range the data exhibit the same trend as for larger times. The evolution in the whole range is therefore uniform, and we find no arguments to attribute the condition of quasistationarity to the shorttime interval. As for the small interval around $t=500$, we also include in Fig. 2 a data set from Ref. 7 , calculated with higher numerical precision (open circles) [18. These data reveal a minimum in $T(t)$ at $t \approx 300$ and confirm the absence of any plateau. Figure 3 shows the data from Fig. 1 for $N=10^{3}$ and $10^{4}$ in linear time scale. Again, we find no evidence of the existence of plateaus or QSS.

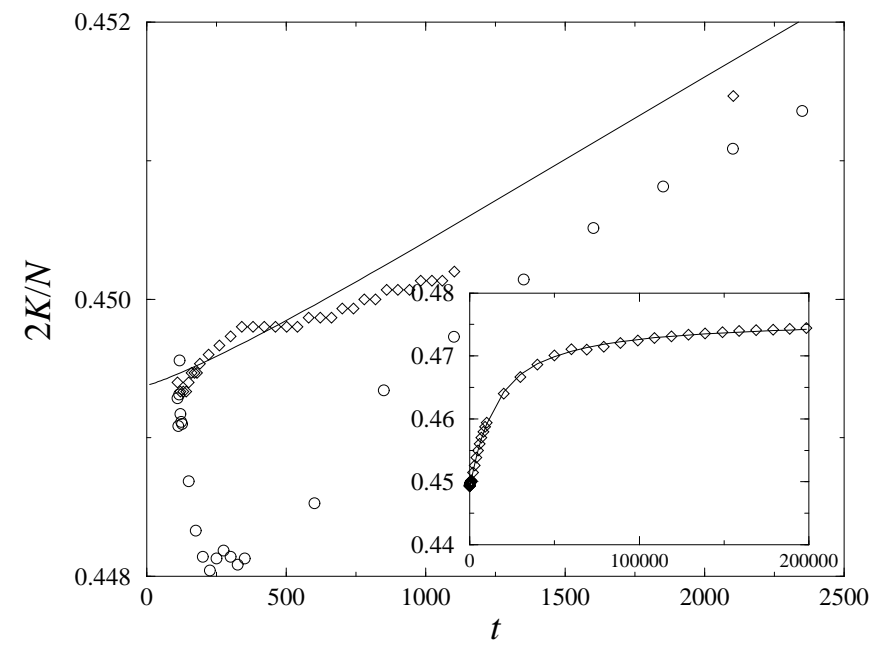

FIG. 2: The same data as in Fig. 1 f for $N=500$ (diamonds), with linear time scale. The inset shows the whole time range. The curve is a Padé-like approximation over the entire range. Open circles are higher-precision results from Ref. 7.

Let us mention that, more indirectly, the observation of anomalous diffusion in the slow-relaxation stage of the HMF model [4 confirms that no special regimes exist within that stage. In fact, superdiffusion in the unfolded $\theta$-space, $\theta \in(-\infty,+\infty)$, which is characterized by an anomalous dispersion law $\left\langle\Delta \theta^{2}\right\rangle \sim t^{\alpha}$, is recorded in the whole slow-relaxation stage with a constant exponent $\alpha \approx 1.4$. The transition to ordinary diffusion $(\alpha=1)$ occurs at the same time as the system reaches canonical equilibrium. The short-time plateau of Fig. 11 and the crossover to canonical equilibrium are not detected by the transport properties of this system.
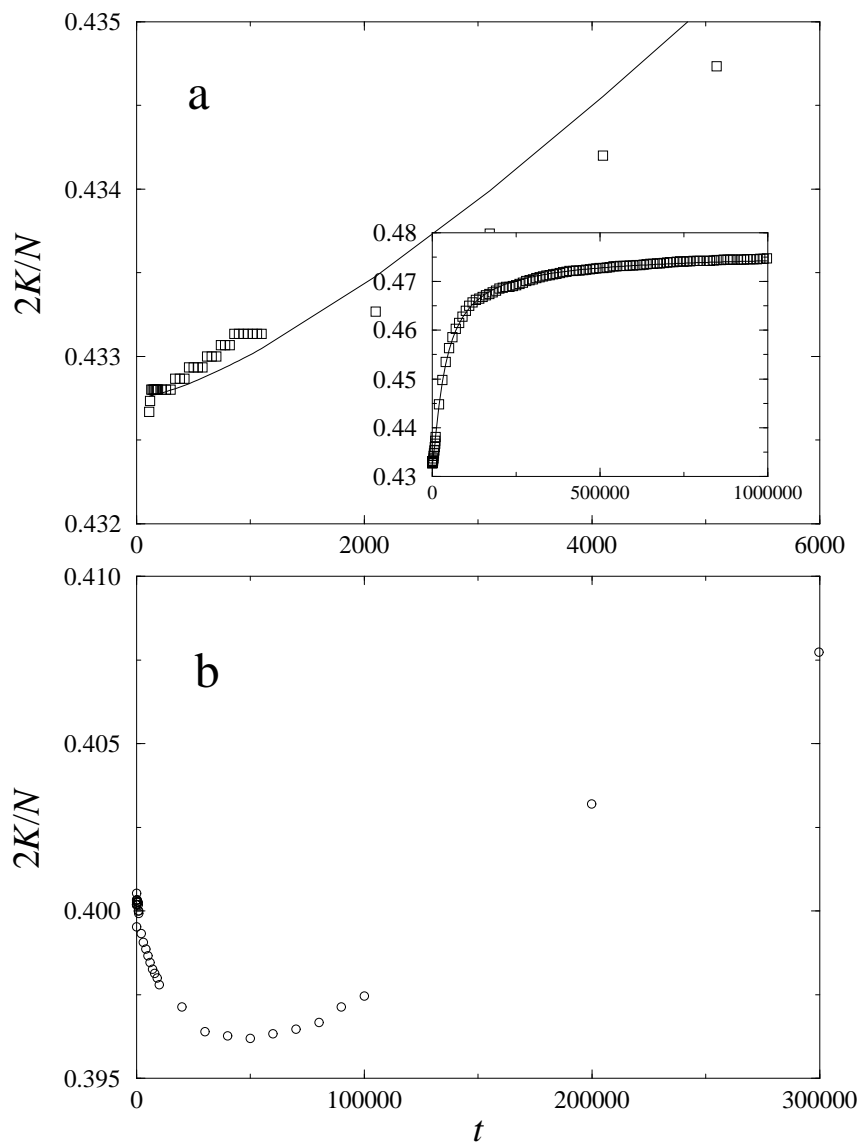

FIG. 3: The same data as in Fig. 1 for (a) $N=10^{3}$ and (b) $N=10^{4}$, with linear time scale. The inset in (a) shows the whole time range, while the curve is a Padé-like approximation over the entire range.

The data shown in Figs. 2 and 3 exhibit an inconsistency regarding the behavior of the nonequilibrium temperature in the first stage of the slow-relaxation regime. In fact, while in the results for $N=500$ (circles) and for $N=10^{4}$ we observe a well-defined minimum in $T(t)$, low-precision data for $N=500$ (diamonds) and results for $N=10^{3}$ do not display such minimum. To clarify this aspect of the evolution, we have performed extensive numerical calculations for the same value of the energy, $U=0.69$, maintaining the relative error in the total energy conservation around $\Delta E / E \sim 10^{-3}$, which makes it possible to average over many realizations of the water-bag initial conditions $\left(5 \times 10^{4}\right.$ for $N=500$ to $10^{3}$ for $\left.N=3000\right)$. Our results for the nonequilibrium temperature $T(t)$ are shown in Fig. 4 , as a function of the rescaled time $t / N$. Each dot has been obtained as an average in time, over $0.1 N$ to $0.2 N$ time units. The arrows indicate, for $N=1000$ and 3000, the typical 
dispersion in the values of $T(t)$ after averaging over the whole set of realizations. Fluctuations are indeed large, as expected near a phase transition and due to the highly chaotic HMF dynamics [2, 3], which makes it difficult to obtain reliable numerical results. In any case, our numerical calculations consistently confirm the existence of a minimum in the nonequilibrium temperature, and the subsequent steady growth of $T(t)$. The inset shows the position of the minimum, $t_{\min }$, as a function of $N$, including also the value for $N=10^{4}$ taken from the data of Fig. 3(b). The straight line corresponds to a power-law fitting, $t_{\min } \propto N^{\nu}$, which yields $\nu=1.7 \pm 0.1$. Though the plot spans less than two decades in $N$, the fact that $t_{\text {min }}$ grows faster than linearly with the system size is clear.

Meanwhile, no plateau is observed, at least, of the kind shown in Fig. 1. A plateau may of course be compulsively ascribed to the zone around the minimum of $T(t)$ at $t_{\text {min }}$, but it would be far from coinciding in nature with the QSS reported by LRT. In fact, although the minimum becomes broader for larger $N$, it also shifts to longer times, which hardly insures that a stationary (nonequilibrium) temperature would be observed in the limit $N \rightarrow \infty$.

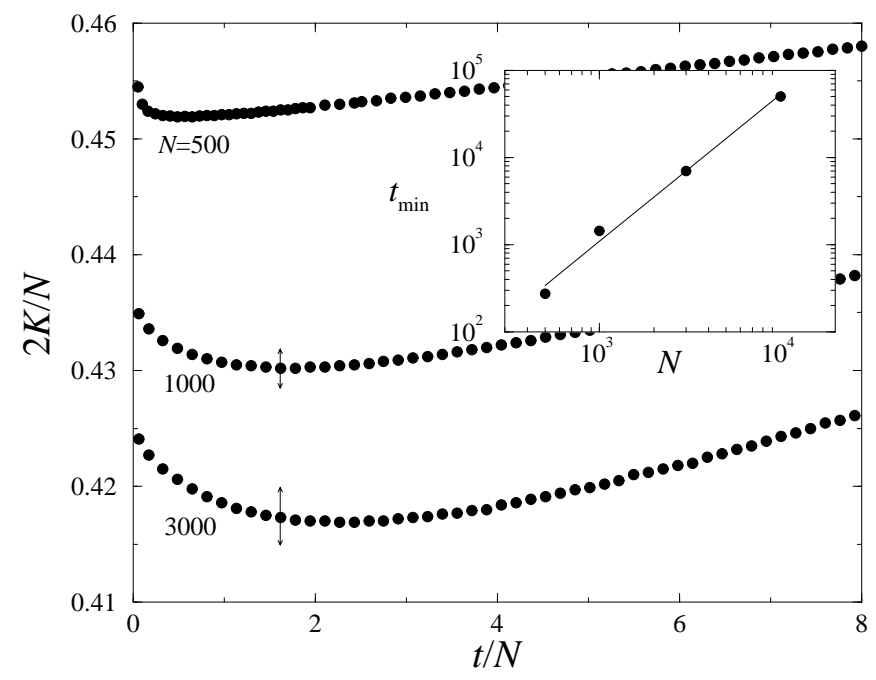

FIG. 4: Nonequilibrium temperature, $T(t)=2 K(t) / N$, for $U=0.69$ and three values of $N$, as a function of the rescaled time $t / N$. The arrows indicate the typical dispersion of $T(t)$ after averaging over a large set of realizations $\left(\sim 10^{3}\right.$ to $\sim 10^{4}$, see text). For $N=500$ the dispersion is negligible in this scale. The inset shows the time of the minimum in $T(t)$ as a function of $N$. The straight line is a power-law fit, with slope 1.7 .

The well-defined minimum of the nonequilibrium temperature $T(t)$ at $t_{\min }$ establishes a quite natural boundary between short-time and long-time dynamical ranges. It is interesting to analyze in more detail the short-time evolution, with emphasis in the angle and momentum distribution of the ensemble in $\mu$-space, where at each time the system is represented by a set of $N$ points in the $(\theta, p)$ plane. One-particle distributions of both angles and momenta have received particular attention in previous work 1 1, 3, 6, 7, so that such representation is useful for comparison. Figure 5 shows four snapshots, at different times, of the $\mu$-space distribution in a single (typical) realization of the HMF model with $N=10^{4}$ rotators, at $U=0.69$. For clarity, the origin of the angle variable, $\theta=0$, has been chosen to coincide in each plot with the phase $\Phi$ of the total magnetization $\mathbf{M}$. In this way, the center of the distribution coincides approximately with the center of the plot. Take into account, however, that $\Phi$ performs, for short times, diffusive-like motion [1]. The WB initial condition would here correspond to a uniform distribution along a vertical segment at $\theta=0$, with $|p|<\sqrt{6 U} \approx 2.03$. As advanced above, we observe a rapid broadening of the distribution in the angle coordinate $\theta$, accompanied by a moderate collapse in $p$. At $t=100$, much of the ensemble has become strongly mixed in $\mu$-space, due to the highly chaotic nature of the dynamics for this value of $U$ [1, 3]. There is however a substantial fraction of the ensemble, represented by the zones where points remain ordered, where the strong correlations imposed by the WB initial condition persist. This fraction is mainly situated near the center of the distribution, in a region of relatively small momentum, but ordered arrays of points are also seen in other zones of $\mu$-space. For these times, thus, the system can be thought of as a mixture of two phases, namely, a strongly mixed, "gaseous" phase and a highly correlated, "condensed" phase.

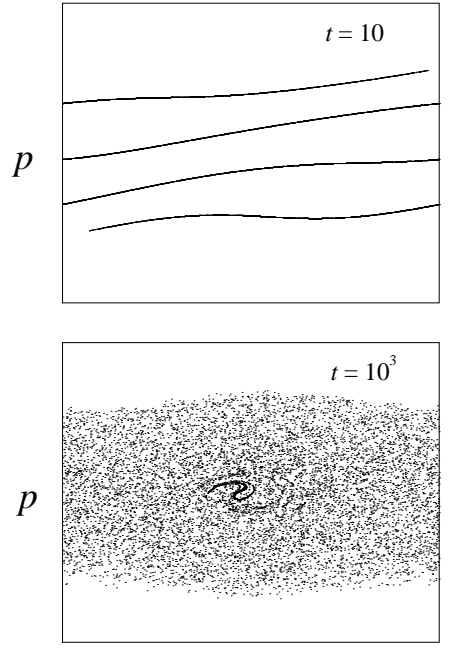

$\theta$
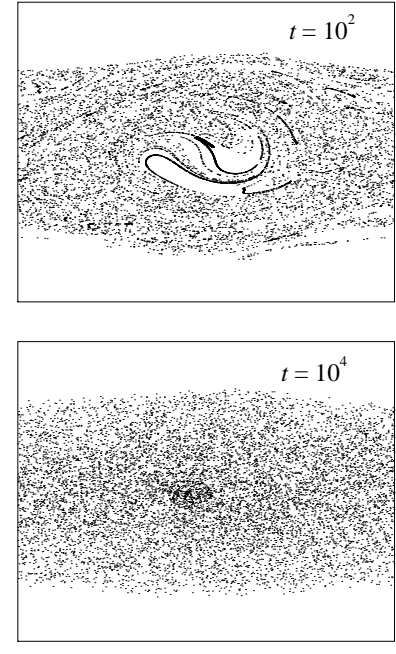

$\theta$
FIG. 5: Snapshots in $\mu$-space at four different times of a single realization of the HMF model with water-bag initial conditions $\left(N=10^{4}, U=0.69\right)$. In the four plots the horizontal and vertical axes span the intervals $(-\pi, \pi)$ and $(-2,2)$, respectively.

The distribution of the condensed phase in $\mu$-space results to strongly depend on the specific realization of the 
WB initial condition. This sensibility to the initial conditions, illustrated in Fig. 6, is again a consequence of the chaotic dynamics that, as time elapses, amplifies the originally small variations between different realizations. An important byproduct of this property, combined with the persistence of the initial correlations, is that the condensed phase is not ergodic, in the sense that averages over realizations of the WB initial condition yield a poor description of the statistical properties over time of any single realization. The lack of ergodicity of the condensed phase makes in turn the whole system nonergodic in this short-time range. An illustration of this overall lack of ergodicity is provided by the short-time evolution of the nonequilibrium temperature. In any single realization, after its initial drop, $T(t)$ displays chaotic oscillations of appreciable amplitude. Due to the sensibility to initial conditions, however, the phases of such oscillations differ between realizations, and the oscillations disappear upon averaging. This observation should warn of the averaging procedures used in previous work [7] to study statistical properties such as the nonequilibrium temperature and the momentum distribution function in the initial regime of kinetic relaxation. The warning applies also to the average data of $T(t)$ shown in our Fig. 1 at the shortest times. Before the nonergodic condensate disappears, in fact, a (standard) statistical approach to the HMF model results to be of limited applicability.
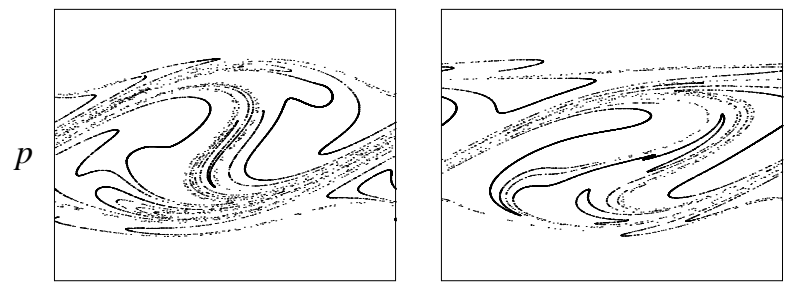

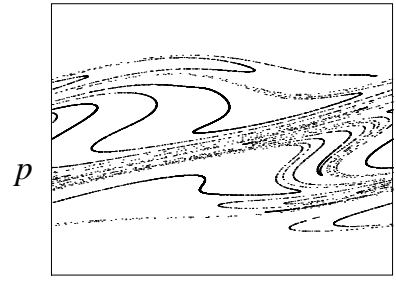

$\theta$

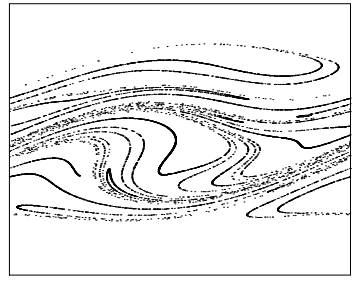

$\theta$
FIG. 6: Snapshots in $\mu$-space at time $t=30$ of four realizations of the HMF model with water-bag initial conditions $\left(N=10^{4}, U=0.69\right)$. Horizontal and vertical scales are as in Fig. 5 .

As the evolution proceeds, particles from the condensate "evaporate" into the gaseous phase [1, 2]. From Fig. 5 we note that, at $t=10^{4}$, only faint traces of the condensed phase remain in the ensemble. We have studied the dynamics of this evaporation process as follows. First of all, we have numbered the rotators in each realization of the HMF model in such a way that, at the initial time, $p_{i}(0)<p_{j}(0)$ for $i<j$. At subsequent times, we have defined the condensate as formed by the rotators $i$ such that the distance to $i+1$ in $\mu$-space, $\delta_{i}=\sqrt{\left(\theta_{i+1}-\theta_{i}\right)^{2}+\left(p_{i+1}-p_{i}\right)^{2}}$, is lower than a given threshold $\delta$. We fix the threshold as the average distance between contiguous pairs at $t=0, \delta=2 \sqrt{6 U} / N$.
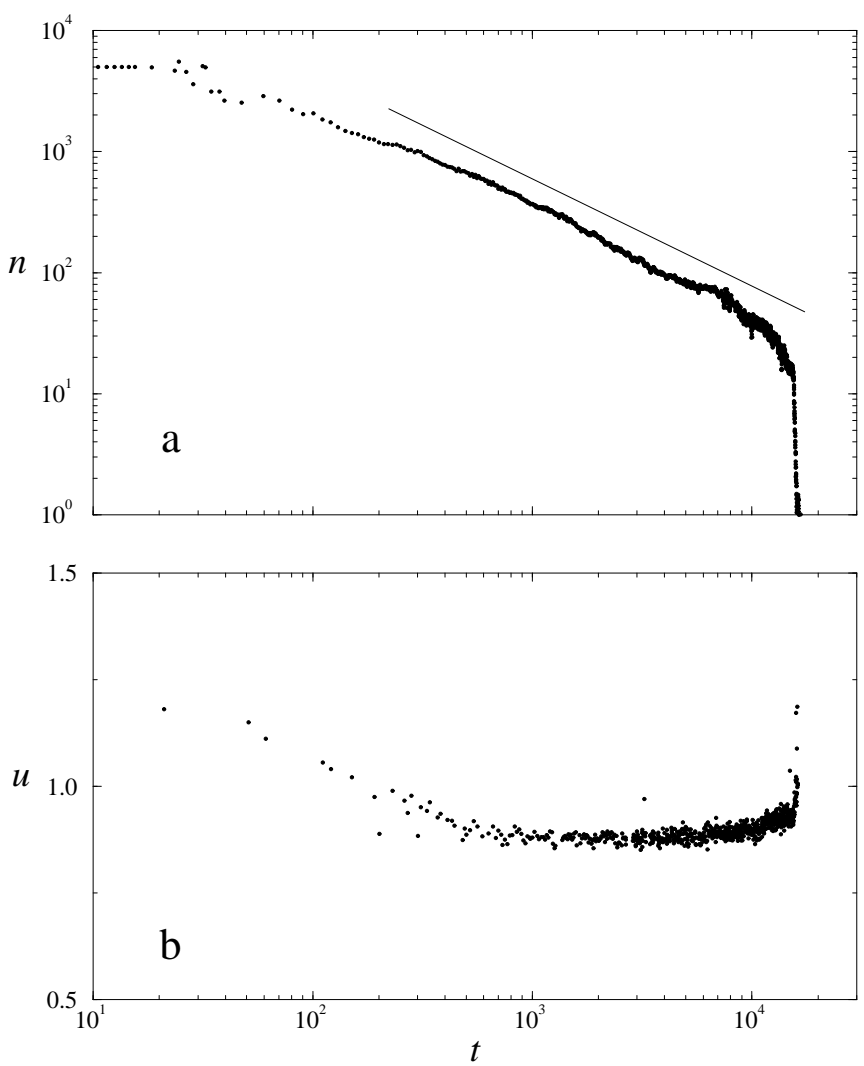

FIG. 7: Evolution of (a) the size of the condensate phase and (b) the average energy per particle in the condensate, for a single realization of the HMF model with $N=10^{4}$ and $U=0.69$. Each dot corresponds to a running average over 100 time units. The straight line in (a) has slope -0.85 .

The number $n$ of rotators in the condensed phase displays considerable oscillations in time. According to the discussion in the previous paragraph, we choose to measure $n$ in a single (typical) realization for $N=10^{4}$ and to average over time intervals. Figure 7 (a) shows the evolution of $n$ averaged each 100 time units. The condensate size decreases steadily, displaying most of the time a power-law decay with a nontrivial exponent, $n \sim t^{-0.85}$. This rather smooth decay is abruptly interrupted by a sudden collapse of the condensate, at $t \approx 1.6 \times 10^{4}$. In Fig. 7(b), we show the evolution of the average energy per particle in the condensate. We see that it varies nonmonotonically with time, though its value is never far from $u=1$. Other realizations for the same value of $N$ confirm that these results are generic. Moreover, they are robust with respect to the definition of the threshold $\delta$. For different values of $N$, realizations in the short-time range show that the collapse of the condensed phase oc- 
curs at a time preceding, but approximately equal to, $t_{\text {min }}$. In other words, when the minimum of the nonequilibrium temperature is attained, the condensate has just evaporated. From then on, the system enters the final stage of its relaxation to canonical equilibrium.

In the long-time range, when the ensemble of rotators has presumably attained an ergodic state, its $\mu$-space configuration can be described statistically, in terms of averages over realizations, by means of a distribution function $f(\theta, p)$. Previous results on the $\mu$-space distribution of the HMF model refer, in all cases, to reduced distribution functions, $f_{\theta}(\theta)$ and $f_{p}(p)$. In Ref. 3, the momentum distribution function is used to illustrate, for $U=0.69$, slow relaxation for long times. For other values of $U$, far from the critical point, both $f_{\theta}$ and $f_{p}$ were compared at moderately long times with the prediction of canonical statistics, showing the relatively fast attainment of canonical equilibrium for such energies. In Refs. 6 and 6, in contrast, deeper significance is attributed to nonequilibrium distribution functionsalways in connection with QSS. There, LRT suggest that $f_{p}$ exhibits the fingerprints of nonextensive thermodynamics, and support this claim showing that the momentum distribution can be acceptably fitted, at low values of $p$, by the power-law distribution derived from Tsallis's nonextensive formulation [11]. Tsallis's formulation, however, predicts the one-particle probability distribution for the energy, and not for the momentum. Now, since the one-particle energy [1]

$$
\epsilon=\frac{p^{2}}{2}+1-M_{x} \sin \theta-M_{y} \cos \theta
$$

depends both on $\theta$ and $p$, statistical descriptions in terms of $\epsilon$ and $p$ are equivalent only if the $\mu$-space distribution is independent of $\theta$, i.e. if the energy distribution function $f_{\epsilon}(\epsilon)$ can be unambiguously given in terms of $f_{p}(p)$. This is not our case for $U<U_{c}$, unfortunately, since the angle distribution is not uniform, as illustrated by our Figs. 5 and 6, and by LRT's figure 3(a) in Ref. 6. Ignoring the angular dependence of the one-particle distribution in $\mu$-space invalidates the identification of $f_{p}$ as an equilibrium distribution derived from a generalized thermodynamical formulation like Tsallis's, which-just as ordinary thermodynamics - yields canonical distributions as functions of the energy.

We have evaluated $f_{\epsilon}$ by means of numerical realizations of the HMF model for $N=10^{4}$. From the energy distribution function we can in turn calculate the energy probability density $P(\epsilon)$, since $f_{\epsilon}(\epsilon)=\rho(\epsilon) P(\epsilon)$, where $\rho(\epsilon)$ is the density of states. The HMF density of states can be expressed in terms of elliptic functions, as

$$
\rho(\epsilon)=\frac{2 \sqrt{2}}{\pi \sqrt{\epsilon-1+M}} K\left(\frac{2 M}{\epsilon-1+M}\right)
$$

for $\epsilon>1+M$ and

$$
\rho(\epsilon)=\frac{2 \sqrt{2}}{\pi \sqrt{\epsilon-1+M}} F\left(\frac{1}{2} \arccos \frac{1-\epsilon}{M}, \frac{2 M}{\epsilon-1+M}\right)
$$

for $1-M<\epsilon<1+M$, where $K$ and $F$ are the complete and incomplete elliptic integrals of the first kind, respectively 19. The energy is bounded from below, at $\epsilon_{\min }=1-M$. Figure 8 shows the results for $P(\epsilon)$ at three times, plotted as a function of $\epsilon-\epsilon_{\min }$. The straight line represents the canonical prediction, $P_{\text {eq }}(\epsilon) \propto \exp (-\epsilon / T)$. Observe that $t=5 \times 10^{4}$ corresponds to the minimum of the nonequilibrium temperature, at the very beginning of the long-time range. For this time we note a considerable deviation from canonical equilibrium, with an abrupt cutoff at $\epsilon-\epsilon_{\min } \approx 1$ preceded by an overpopulated interval. For very low energies, however, the coincidence with the equilibrium distribution is already quite good. For $t=2 \times 10^{5}$ the cutoff persists but it is less abrupt and has shifted to higher energies, while in the low-energy range the distribution is closer to canonical equilibrium. Finally, for $t=10^{6}$, canonical equilibrium is well established in almost the whole range of energies shown in the figure. Deviations of the nonequilibrium temperature with respect to $T_{\text {eq }}$ are therefore to be ascribed to the depleted tail at high energies. From these results, we conclude that in the long-time regime the equilibrium energy distribution of the HMF model with WB initial conditions is built up from low energies, with a depleted high-energy tail that recedes as time elapses.

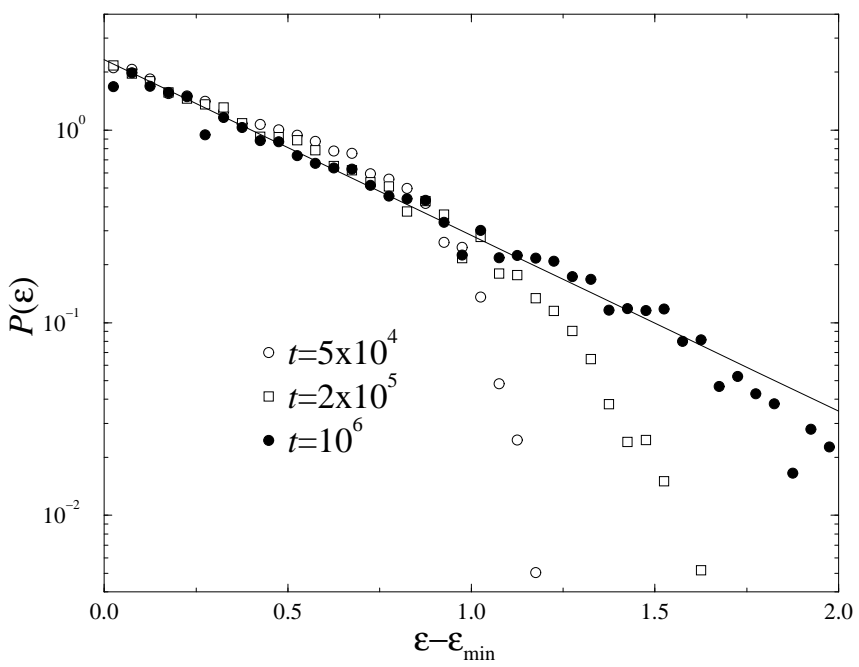

FIG. 8: Probability distribution of energies at three times in the long-time range, for a system of $N=10^{4}$ rotators at $U=$ 0.69 , as a function of $\epsilon-\epsilon_{\min }$. The straight line stands for the exponential canonical prediction with temperature $T=0.476$.

We remark that LRT have assigned a distribution function to states which are clearly in the short-time range. In that range the system is still strongly nonergodic and, as discussed above, such statistical description is therefore of very limited significance. Their best fitting, for $N=10^{5}$ at $t=1200$, corresponds in fact to a state where probably most of the ensemble is still in the condensed phase. Moreover, they compare results for differ- 
ent system sizes at the same time [see their figure 2(c) in Ref. [6], disregarding the scaling laws of time scales with the size, which threatens the validity of their evaluation of the fitting quality as a function of $N$ [their figure $2(\mathrm{~d})$ ]. Finally, we mention that the abrupt drop of $f_{p}$ for large momenta - also seen at larger times in our results for $P(\epsilon)$, Fig. 8 eludes any description based on Tsallis's thermodynamics. The artificial cutoff imposed by LRT to their fitting of $f_{p}$, aimed at mimicking its abrupt drop, can hardly find any consistent justification within such formalism. We conclude that no evidence supports the association of nonequilibrium HMF states with generalized thermodynamical equilibria.

In summary, we have reexamined the slow-relaxation dynamics of the Hamiltonian mean-field model, when the evolution starts from water-bag initial conditions. First, we have revised the evidence that would support the existence of quasistationary states in the nonequilibrium dynamics of the model. In that regard, we have shown that the plateaus in the temporal evolution of the nonequilibrium temperature $T(t)$ [6, 7] are a mere artifact of the use of a logarithmic scale for the time axis in the plot of $T(t)$. Moreover, a detailed analysis of the evolution of the nonequilibrium temperature shows that the dynamics can be decomposed into two well-defined dynamical regimes: a short-time range characterized by the presence of a condensed phase, in which the system preserves strong memory of the initial conditions, and a long-time range, in which the condensed phase has evaporated and the system slowly relaxes toward equilibrium. A natural boundary between these two regimes is given by the minimum reached by the nonequilibrium temperature, which recedes toward larger times as the system size grows. The numerical evaluation of the probability density function of the one-particle energy in the long-time range demonstrates that the system is slowly relaxing to the Boltzmann distribution, and does not support any connection with the generalized equilibrium distributions derived from Tsallis's nonextensive formalism.
It must be stressed that neither previous work nor our present results elucidate the nature of the HMF evolution in the thermodynamical limit $N \rightarrow \infty$. The existence of different dynamical regimes, with different scaling laws in terms of $N$, makes an extrapolation from the available numerical results extremely uncertain. The possibility of performing significant, extensive numerical realizations of this model with current computational facilities seems to be restricted to ensembles of, at most, $N \sim 10^{4}$ elements. Numerical realizations for much larger systems may be necessary in order to attain a meaningful approach to the thermodynamical limit.

The origin of slow dynamics in the Hamiltonian meanfield model, also revealed by the study of aging phenomena and strong memory effects in the long-time range [9], remains to be explained as well. A plausible argument [8] may perhaps arise from the study of the role of the state of zero magnetization, which for $U<U_{c}$ is thermodynamically unstable, and its connection with critical slowing down [2] - a connection which has often been invoked but not yet investigated. Revealing the basic mechanisms of slow relaxation in the Hamiltonian mean-field model should definitely clarify its relation to systems with some degree of structural disorder or frustration, such as glasses, that also exhibit slow-dynamics features. In turn, this would contribute to a unified view on nonequilibrium phenomena in systems with many degrees of freedom.

\section{Acknowledgments}

We thank S. Ruffo for his motivating remarks on the HMF model, and C. Tsallis for comments on his own work. D. H. Z. acknowledges hospitality at the Abdus Salam International Centre for Theoretical Physics (Trieste, Italy), where this work was partially carried out.
[1] M. Antoni and S. Ruffo, Phys. Rev. E 52, 2361 (1995).

[2] V. Latora, A. Rapisarda and S. Ruffo, Phys. Rev. Lett. 80, 692 (1998).

[3] V. Latora, A. Rapisarda and S. Ruffo, Physica D 131, 38 (1999).

[4] V. Latora, A. Rapisarda and S. Ruffo, Phys. Rev. Lett. 83, 2104 (1999).

[5] F. Tamarit and C. Anteneodo, Phys. Rev. Lett. 84, 208 (2000).

[6] V. Latora, A. Rapisarda and C. Tsallis, Phys. Rev. E 64, 056134 (2001).

[7] V. Latora, A. Rapisarda and C. Tsallis, Physica A 305, 129 (2002).

[8] M. Antoni, H. Hinrichsen and S. Ruffo, Chaos, Solitons and Fractals 13, 393 (2002).

[9] M. Montemurro, F. Tamarit and C. Anteneodo, condmat/0205355
[10] C. Tsallis, Physica A 302, 187 (2001).

[11] C. Tsallis, Physica A 221, 277 (1995).

[12] T. Tsuchiya, T. Konishi, and N. Gouda, Phys. Rev. E 50, 2607 (1994).

[13] Note that the definitions of the water-bag initial condition respectively used for gravitational systems 12] and for the HMF model are substantially different. To our knowledge, the possible consequences of this difference have not been discussed in the literature.

[14] Lj. Milanović, H. A. Posch, and W. Thirring, Phys. Rev. E 57, 2763 (1998).

[15] The analysis of $S(t)$-like most studies of the HMF dynamics - disregards the short initial stage of kinetic relaxation.

[16] Data obtained from the version of Ref. 6 at the public eprint archive http://arXiv.org, cond-mat/010354d. The original figure also includes data for $N=10^{5}$, but they 
do not span a sufficiently wide temporal range.

[17] For instance, the function $T(t)=T_{1}+\left(T_{0}-\right.$ $\left.T_{1}\right) \exp (-t / \tau)$, whose derivatives are all monotonic functions, acquires an inflection point at $t=\tau$ if plotted as a function of $\log t$.
[18] Data obtained from the version of Ref. 7 at the public e-print archive http://arXiv.org, cond-mat/0109056.

[19] M. Abramowitz and I. Stegun, Handbook of Mathematical Functions (Dover, New York, 1972). 